\title{
Urgent-start peritoneal dialysis for patients with end stage renal disease: a 10-year retrospective study
}

\author{
Hongjian Ye ${ }^{1,2 \dagger}$, Xiao Yang ${ }^{1,2+}$, Chunyan $\mathrm{Yi}^{1,2}$, Qunying Guo ${ }^{1,2}$, Yafang $\mathrm{Li}^{1,2}$, Qiongqiong Yang ${ }^{1,2}$, Wei Chen ${ }^{1,2}$, \\ Haiping Mao ${ }^{1,2}$, Jianbo Li ${ }^{1,2}$, Yagui Qiu ${ }^{1,2}$, Xunhua Zheng ${ }^{1,2}$, Dihua Zhang ${ }^{1,2}$, Jianxiong Lin ${ }^{1,2}$, Zhijian Li ${ }^{1,2}$, \\ Zongpei Jiang ${ }^{1,2}$, Fengxian Huang ${ }^{1,2}$ and Xueqing $Y^{1,2^{*}}$ (iD
}

\begin{abstract}
Background: Urgent-start peritoneal dialysis (PD) can help patients with end-stage renal diseases (ESRD) that are referred late to dialysis. However, catheter patency and related complications of urgent-start PD have not been thoroughly clarified. We investigated the clinical outcomes of urgent-start PD in a Chinese cohort.

Methods: We enrolled ESRD patients who received urgent-start PD (starting PD within 14 days after catheter insertion) in our center from January 1, 2006 to December 31, 2014, and followed them up for 10 years. The primary outcome was catheter failure. Secondary outcomes included short-term and long-term complications related to urgent-start PD.

Results: Totally 2059 patients (58.9\% male, mean age $47.6 \pm 15.9$ years) were enrolled. Few perioperative complications were observed, including significant hemorrhage $(n=3,0.1 \%)$ and bowel perforation $(n=0)$. Early peritonitis occurred in $24(1.2 \%)$ patients $(0.28$ episodes per patient-year). Within the first month after catheter insertion, functional catheter malfunction occurred in 85 (4.1\%) patients, and abdominal wall complications (including hernia, hydrothorax, hydrocele, and leakage) in 36 (1.7\%) patients. During a median 36.5 (17.7-61.4) months of follow-up, 75 (3.6\%) patients experienced catheter failure, and 291 (14.1\%) had death-censoring technique failure. At the end of 1-month, 1 -year, 3-year, and 5-year, catheter patency rate was 97.6, 96.4, 96.2, 96.2\%; and technique survival rate was 99.5, 97.0, 90.3, 82.7\%, respectively. After adjusting for confounders, every 5year increase in age was associated with 19\% decrease of risk for catheter failure (hazard ratio [HR]: 0.81, 95\% confidence interval [Cl]: 0.73-0.89). Male sex (HR: 1.43, 95\% Cl: 1.00-2.04), diabetic nephropathy (HR: 1.56, 95\% Cl: 1.08-2.25) and low hemoglobin levels (HR: 0.89, 95\% Cl: 0.81-0.98) were independent risk factors for abdominal wall complications.
\end{abstract}

Conclusions: Urgent-start PD is a safe and efficacious option for unplanned ESRD patients. A well-trained PD team, a standardized catheter insertion procedure by experienced nephrologists, and a carefully designed initial PD prescription as well as comprehensive follow-up care, might be essential for the successful urgent-start PD program.

Keywords: End stage renal disease, Peritoneal dialysis, Urgent-start peritoneal dialysis, Technique survival, Catheter patency, Complications

\footnotetext{
* Correspondence: yuxq@mail.sysu.edu.cn

${ }^{\dagger}$ Hongjian Ye and Xiao Yang have contributed equally to this work

'Department of Nephrology, The First Affiliated Hospital, Sun Yat-sen

University, 58th, Zhongshan Road II, Guangzhou 510080, China

${ }^{2}$ Key Laboratory of Nephrology, Ministry of Health and Guangdong Province,

Guangzhou 510080, Guangdong, China
}

(c) The Author(s). 2019 Open Access This article is distributed under the terms of the Creative Commons Attribution 4.0 International License (http://creativecommons.org/licenses/by/4.0/), which permits unrestricted use, distribution, and reproduction in any medium, provided you give appropriate credit to the original author(s) and the source, provide a link to the Creative Commons license, and indicate if changes were made. The Creative Commons Public Domain Dedication waiver (http://creativecommons.org/publicdomain/zero/1.0/) applies to the data made available in this article, unless otherwise stated. 


\section{Background}

End stage renal disease (ESRD) has become a heavy burden to public health worldwide. According to the 2017 United States Renal Data System (USRDS) report, the crude incidence of ESRD diagnoses was 378 per million/ year. Suprisingly, even with regular nephrology followup, more than $60 \%$ of the new diagnosed ESRD patients do not have clear plans when they begin renal replacement therapy, which prodispose them to a need for urgent dialysis [1]. China is limited in some healthcare resources, particularly in rural and remote areas [2]. A cross-sectional survey in China showed the overall prevalence of chronic kidney disease (CKD) to be $10.8 \%$, with wide regional variations [3]. As most CKD patients are referred late to dialysis, unplanned dialysis is common in China [4, 5]. Moreover, as hemodialysis (HD) is unavailable in most rural or remote areas in China, peritoneal dialysis (PD) is the only option [6].

As a home-based renal replacement modality, PD has been increasingly used by ESRD patients. By the end of 2016, China had 74,138 registered patients on PD [7]. Sterile surgical technique is the most frequently used method for PD catheter placement in our region. Previous guidelines recommended that catheter insertion should be performed at least 2 weeks before starting PD [8, 9]. However, it is unrealistic for these unplanned dialysis patients to wait for a two-week's period before initiating PD therapy. Urgent-start PD has been suggested to be an effective approach to prompt initiation of PD after catheter insertion, may avoid increased risk of central venous catheterrelated complications, including bacteremia, central venous stenosis, and thrombosis associated with temporary use of $\mathrm{HD}[5,10]$. However, the possibility of adverse effects from urgent-start PD, such as catheter dysfunction, fluid leakage, and early infection, was a major impediment to its wider use $[8,11]$. Although several studies have reported complications related to urgent-start PD, their evidence was relatively weak due to limited sample size and regional differences $[5,11-16]$.

In our center, almost all patients with ESRD experienced urgent catheter insertion and immediate initiation of PD therapy. We therefore investigated the prevalence of complications and outcomes of urgent-start PD for these patients.

\section{Methods}

\section{Study population}

We retrospectively enrolled all patients treated at our hospital from January 1, 2006 to December 31, 2014. All patients satisfied the following inclusion criteria: (1) diagnosed with ESRD; (2) Tenckhoff catheters were inserted with sterile surgical technique by nephrologists; and (3) initiated PD therapy within 14 days after catheter insertion.

\section{Urgent-start PD program}

Figure 1 shows the flow chart of the urgent-start PD program, which was defined as starting PD within 14 days after catheter insertion [16]. ESRD Patients who chose PD were placed double-cuff Tenckhoff catheters with open laparotomy technique by experienced nephrologists. A double purse-string suture technique on the posterior rectus sheath and parietal peritoneum was used for catheter fixation and prevention of leakage (Fig. 2). The deep cuff was placed into the rectus abdominis muscle. Intravenous cefuroxime or cefazolin was used as a prophylactic antibiotic $30 \mathrm{~min}$ before catheterization, unless the patient was allergic to penicillin. For those allergic to penicillin, we used single-dose ciprofloxacin for antibiotic prophylaxis. Intermittent peritoneal dialysis (IPD) treatment was initiated immediately after catheter insertion with a dialysate volume of $500 \mathrm{ml}$ per dwell for $1 \mathrm{~h}$ ( 8 cycles per day) in first day at the supine position, then $650 \mathrm{ml}$ per dwell for $1 \mathrm{~h}(9 \mathrm{cy}-$ cles per day) in the next 1-2 days, and gradually increased to $2000 \mathrm{ml}$ or the maximum tolerable volume within 8 to 10 days. According to the program, the IPD regime took about 6-9 h each day. Patients then began continuous ambulatory peritoneal dialysis (CAPD) (Fig. 1). A professional nursing team managed the IPD dialysate exchanges. Patients and their caregivers received a standard training program within 5-7 days after

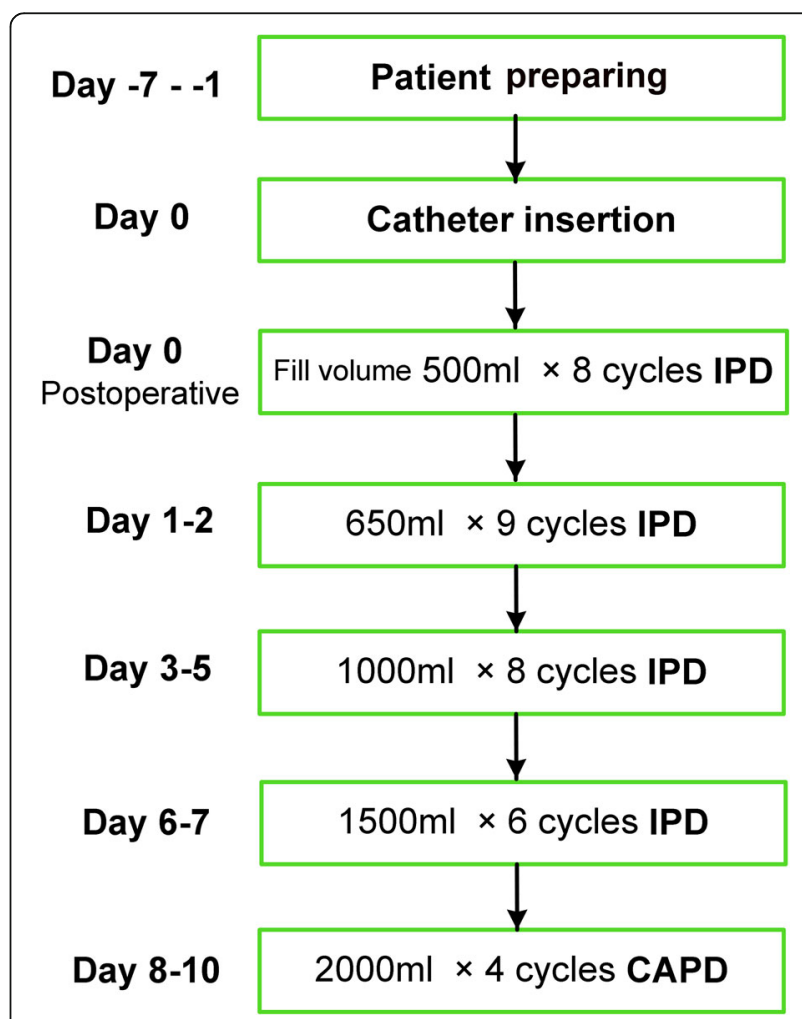

Fig. 1 The flow chart of the urgent-start PD program 


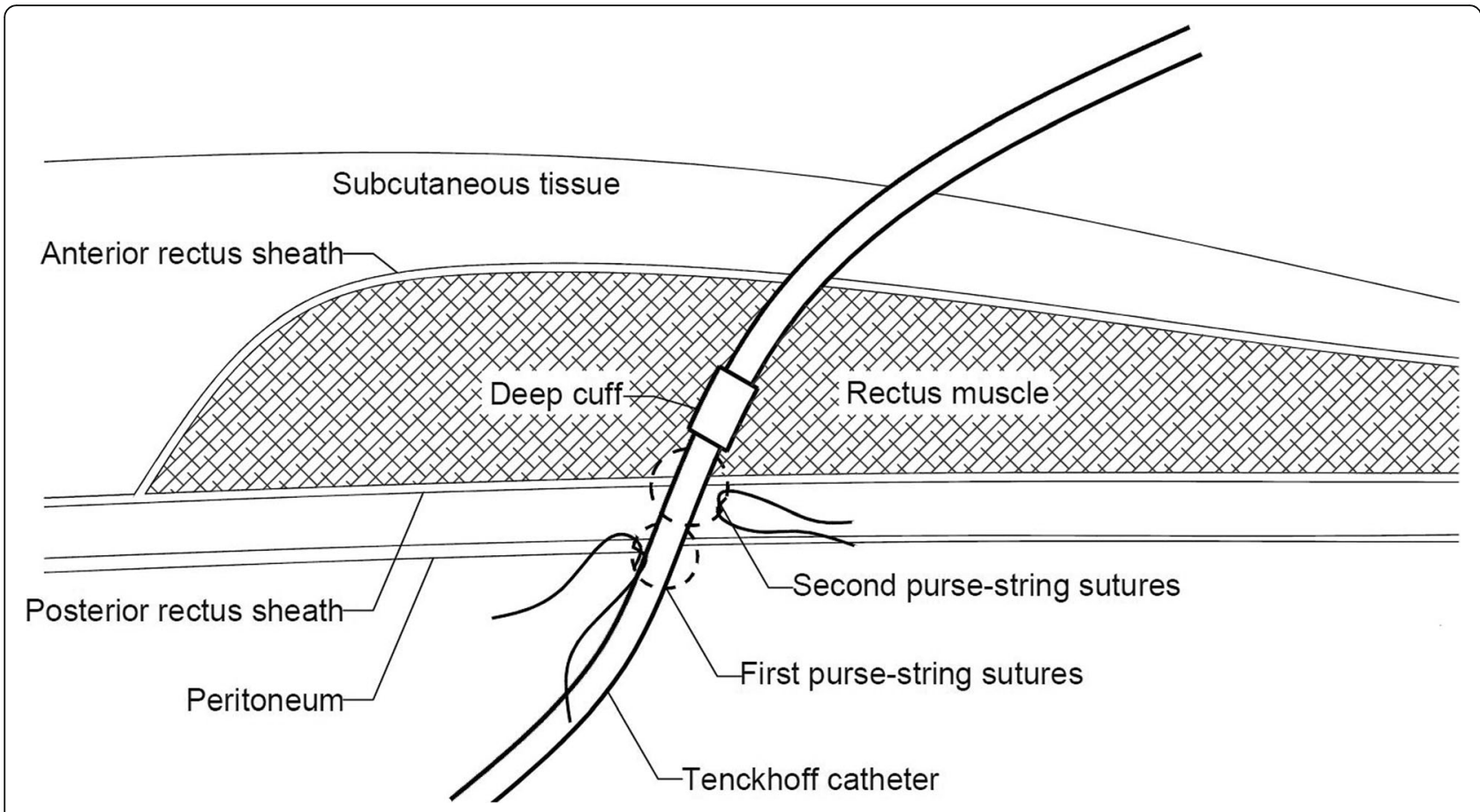

Fig. 2 A schematic diagram of catheterization. A double purse-string sutures technique was used for the catheter fixation and prevention of leakage

catheterization. Post-discharged PD patients were managed according to our follow-up program [17].

\section{Baseline demographic and laboratory data, and study outcomes}

Demographic and clinical data, including age, sex, causes of renal diseases, comorbidity conditions, history of abdominal surgery, body mass index (BMI), and body surface area (BSA) were collected before catheterization. Laboratory data were also evaluated at the baseline. Residual renal function at the initiation of PD was estimated by the CKD-EPI equation [18]. We followed up the patients until any cause of dropping out of PD (death, change to $\mathrm{HD}$, kidney transplantation, loss to follow-up) or December 31, 2016.

The primary outcome was catheter failure, which was defined as functional catheter problems that required catheter manipulation or replacement, or lead to technique failure. Catheter patency was defined as continuation of PD without any catheter manipulation or surgical intervention [9]. Secondary outcomes included deathcensoring technique failure, functional catheter problems, and other urgent-start PD-related complications. Deathcensoring technique failure was defined as transferring to HD from any cause; death, kidney transplantation, loss to follow up, transfer to other centers, and recovery of renal function were considered as censoring events [19]. Functional catheter problems included any troublesome inflow of dialysate and/or outflow of dialysate that possibly needed surgical intervention [20]. Omental wrap was diagnosed by opening procedures in this study. Short-term and long-term urgent-start PD-related complications, such as perioperative complications (including significant hemorrhage requiring transfusion or surgical intervention, bowel perforation), early infections (exit-site infections and peritonitis within 2 weeks after catheter insertion), and abdominal wall complications (including pericatheter leakage, subcutaneous leakage, hernia, hydrothorax, and hydrocele) [9], were recorded during the follow-up period. A PD team of three nephrologists reviewed individual medical records and identified causes for catheter failure, functional catheter problems, or technique failure.

\section{Management of functional catheter problems and abdominal wall complications}

Generally, a patient with functional catheter problem would receive an abdominal X-ray examination to identify whether the catheter had shifted. The drainage bag with dialysate was also examined to find any underlying cause for catheter obstruction. All these patients would initially receive conservative treatments. If the problem was unresolved, surgical interventions such as catheter manipulation, revision, or replacement, were conducted in the case of patient informed consent (Fig. 3).

Patients with abdominal wall complications would receive decreased infused volume, or temporarily suspended 
PD, or temporary HD therapy to allow for the possibility of natural repair (such as scrotal edema, pericatheter leakage, and subcutaneous leakage). If the problems were unresolved, the patients would receive surgical manipulation or transfer to permanent HD, as appropriate.

\section{Statistical analysis}

We expressed the results as mean \pm standard deviation (SD), frequencies (percentages),, and median (interquartile range $[\mathrm{IQR}]$ ) according to the types of variables. The independent sample $t$-test was used for normally distributed continuous variables. The Mann-Whitney U-test was used to compare non-normal distributed continuous variables. For categorical variables, the Chi-square test was used. We apply Kaplan-Meier analysis to evaluate the catheter survival and technique survival. The Cox

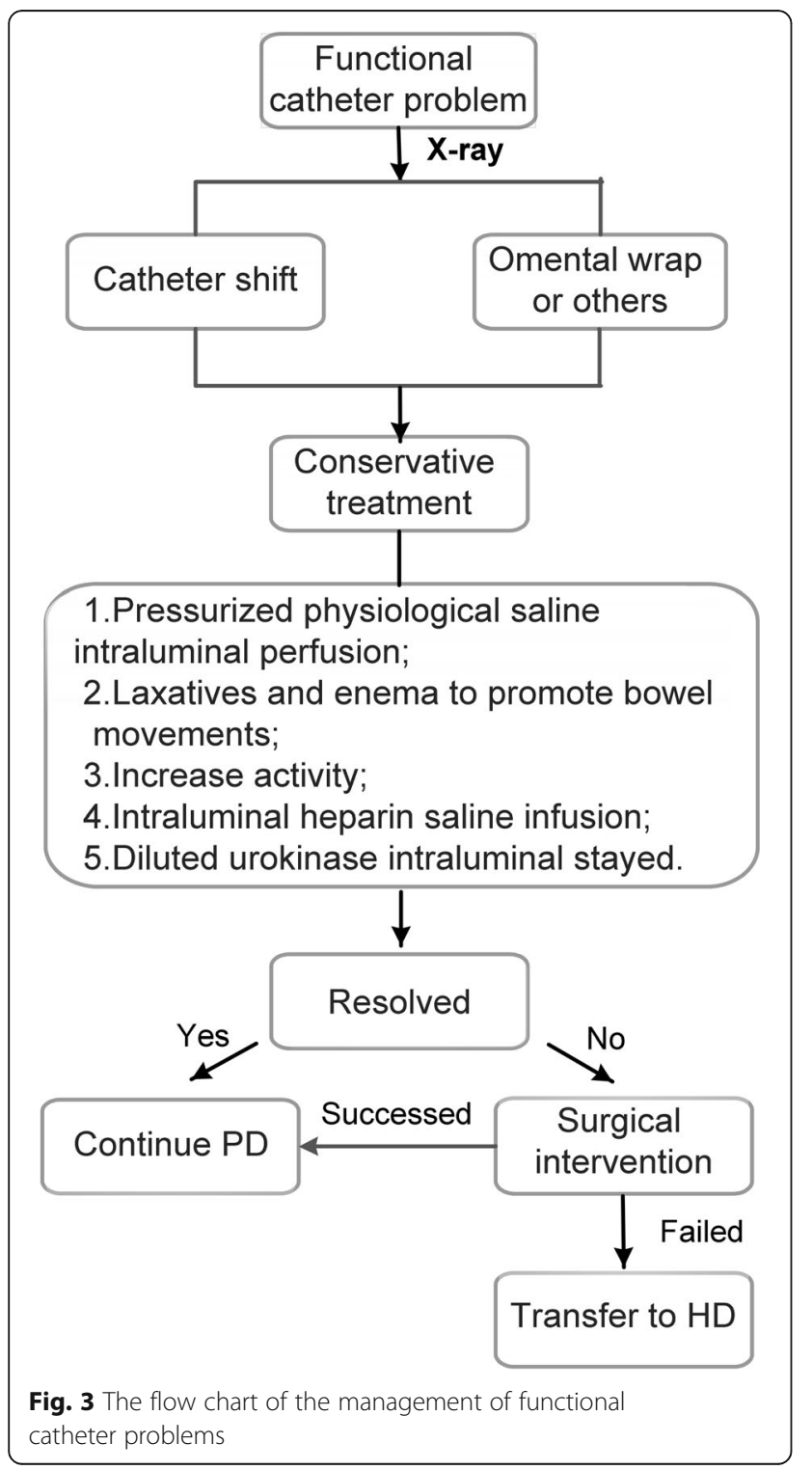

proportional hazards regression models were used to explore risk factors associated with catheter failure and abdominal wall complications. Data were analyzed with SPSS software (version 19.0). $P<0.05$ was considered statistically significant.

\section{Results}

\section{Patient characteristics}

We included 2059 PD patients who met the inclusion criteria at our center: almost all patients initiated PD therapy immediately after catheter insertion and only a few patients who had weakness of peritoneum and rectus abdominis sheath would delay several days (within 14 days after catheter insertion) to start dialysis. Their mean age was $47.6 \pm 15.9$ years; 1212 (58.9\%) patients were male. The most common cause of ESRD was chronic glomerulonephritis (58.8\%), followed by diabetic nephropathy (21.8\%) (Table 1). All the patients were inserted with Tenckhoff catheters using sterile open surgery by experienced nephrologists. Coiled catheters only accounted for $7.4 \%$, the rest were straight catheters. Generally, patients started IPD treatment immediately after catheter insertion with infused dialysis volume gradually increasing to $2000 \mathrm{ml}$ or the maximum tolerable volume, and then started on the regular CAPD regimen as described in Fig. 1.

\section{The prevalence of functional catheter problems and catheter failure}

During a median of 36.5 months (IQR: 17.7-61.4 months) of follow-up, 156 (7.6\%) patients experienced functional catheter problems. Among them, 28.2\% events occurred within 7 days after catheter insertion, $12.2 \%$ occurred between 8 and 14 days, $14.1 \%$ occurred between 15 days and 1 month, and 32.1\% occurred between 1 month and 1 year. The distribution of onset time for catheter failure was similar (Fig. 4). According to clinical and intraoperative assessments, $60.9 \%$ of the functional catheter problems were caused by catheter shift, $17.9 \%$ caused by omental wrap, and $19.9 \%$ from unknown causes. Similarly, catheter shift (65.2\%) was the leading cause for catheter failure; whereas omental wrap accounted for a higher proportion of causes for catheter failure at $32.0 \%$, and unknown causes were only $2.7 \%$.

All 156 patients firstly received series of conservative treatments. Among them, 81 (51.9\%) catheters were rescued and $75(48.1 \%)$ patients had catheter failure. Catheter survival rate was 97.6, 96.4, 96.2, 96.2, and 96.2\%, respectively at the end of 1-month, 1-year, 3-year, 5-year, and 10-year. The successful rescue rate for catheters was 41.3\% for events occurring within 14 days after insertion, $54 \%$ for events occurring between 1 month and 1 year, and $85.7 \%$ for events occurring after the first year; and was highest in patients with catheter obstruction $(100 \%)$ 
Table 1 Demographic characteristics and clinical data

\begin{tabular}{|c|c|c|c|c|}
\hline Variables & Patients $(n=2059)$ & Catheter failure $(n=75)$ & Catheter patency $(n=1984)$ & $P$ value \\
\hline Age (years) & $47.6 \pm 15.9$ & $37.9 \pm 15.0$ & $47.9 \pm 15.8$ & $<0.001$ \\
\hline Sex (Male, n, \%) & $1212(58.9 \%)$ & $45(60 \%)$ & $1167(58.8 \%)$ & 0.905 \\
\hline $\mathrm{BMI}\left(\mathrm{kg} / \mathrm{m}^{2}\right)$ & $21.5 \pm 3.2$ & $20.7 \pm 3.1$ & $21.6 \pm 3.2$ & 0.020 \\
\hline $\mathrm{BSA}\left(\mathrm{m}^{2}\right)$ & $1.59 \pm 0.17$ & $1.56 \pm 0.15$ & $1.58 \pm 0.17$ & 0.237 \\
\hline Primary renal disease $(\mathrm{n}, \%)$ & & & & 0.905 \\
\hline Glomerulonephritis & $1204(58.5 \%)$ & $46(61.3 \%)$ & $1158(58.4 \%)$ & - \\
\hline Diabetic nephropathy & $449(21.8 \%)$ & $14(18.7 \%)$ & $435(21.9 \%)$ & - \\
\hline Hypertensive nephropathy & $151(7.3 \%)$ & $5(6.7 \%)$ & $146(7.4 \%)$ & - \\
\hline Others & $255(12.4)$ & $10(13.3 \%)$ & $245(12.3 \%)$ & - \\
\hline Diabetic nephropathy (n, \%) & $449(21.8 \%)$ & $14(18.7 \%)$ & $435(21.9 \%)$ & 0.502 \\
\hline Type of catheter (coiled VS. straingt)(n, \%) & $152(7.4 \%)$ & $11(14.7 \%)$ & $141(7.1 \%)$ & 0.014 \\
\hline History of abdominal surgery $(n, \%)$ & $149(7.2 \%)$ & $8(10.7 \%)$ & $141(7.1 \%)$ & 0.252 \\
\hline Serum creatinine (mg/dL) & $9.7(7.7-12.2)$ & $9.7(7.7-12.1)$ & $10.7(8.4-13.7)$ & 0.024 \\
\hline eGFR $\left(\mathrm{ml} / \mathrm{min} / 1.73 \mathrm{~m}^{2}\right)$ & $5.0(3.9-6.6)$ & $5.0(3.9-6.6)$ & $4.7(3.5-6.8)$ & 0.384 \\
\hline Hemoglobin (g/dL) & $8.1 \pm 1.9$ & $7.8 \pm 1.5$ & $8.1 \pm 1.9$ & 0.138 \\
\hline Serum albumin (g/dL) & $3.5 \pm 0.5$ & $3.5 \pm 0.6$ & $3.5 \pm 0.5$ & 0.929 \\
\hline
\end{tabular}

NOTE. Values expressed as mean $\pm \mathrm{SD}$, median (interquartile range), or number (percent); Abbreviations: $B M I$ body mass index, $B S A$ body surface area, $e G F R$ estimated glomerular filtration rate. $\mathrm{SI}$ units conversions: serum creatinine, $1 \mathrm{mg} / \mathrm{dL}=88.4 \mu \mathrm{mol} / \mathrm{L} ;$ hemoglobin, $\mathrm{g} / \mathrm{dL}=1 / 10 \mathrm{~g} / \mathrm{L} ; \mathrm{serum}$ albumin, $\mathrm{g} / \mathrm{dL}=1 / 10 \mathrm{~g} / \mathrm{L}$.

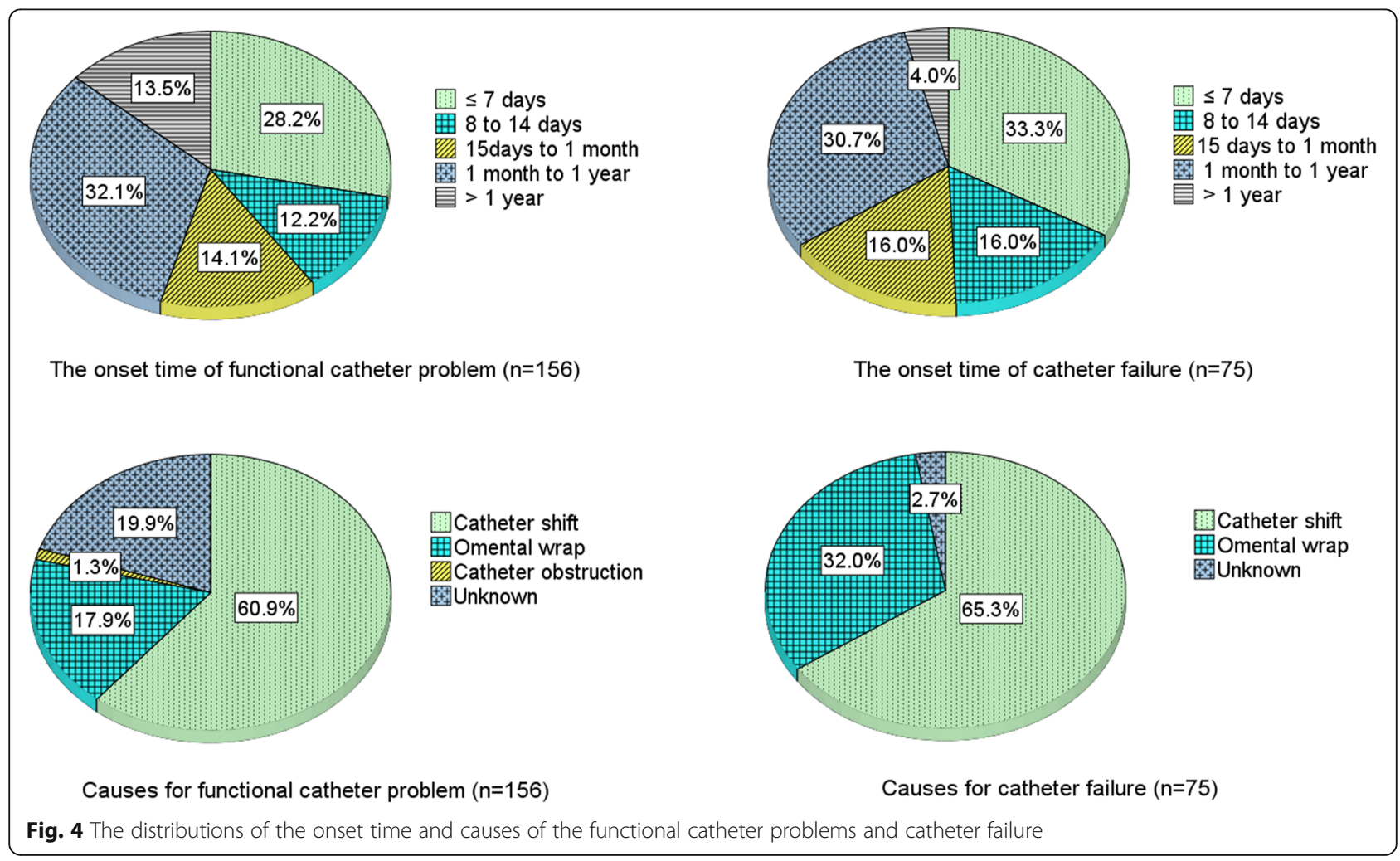


or unknown causes (93.5\%), 48.4\% in cases of catheter shift, and lowest (14.3\%) in cases of omental wrap.

\section{Risk factors for catheter failure}

Compared with patients with catheter patency, those with catheter failure were significantly younger (37.9 \pm 15.0 years vs. $47.9 \pm 15.8$ years), had lower BMI (20.7 \pm $3.1 \mathrm{~kg} / \mathrm{m}^{2}$ vs. $\left.21.6 \pm 3.2 \mathrm{~kg} / \mathrm{m}^{2}\right)$, and lower serum creatinine (9.7 [IQR:7.7-12.1] mg/dL vs. 10.7 [IQR: 8.4-13.7] $\mathrm{mg} / \mathrm{dL}$ ). Patients catheterized with coiled catheters had a significantly higher incidence of catheter failure than those with straight catheters $(14.7 \%$ vs $3.4 \%, P=0.014$; Table 1). In the univariate Cox regression model, baseline younger age, lower BMI, and higher serum creatinine were risk factors for catheter failure. After adjustment for confounders, the multivariate Cox model shown that every 5 years increase in age was associated with 19\% decrease of risk (hazard ratio [HR]: 0.81, 95\%CI: 0.73-0.89) for catheter failure. Similar associations between these risk factors and functional catheter problems were also observed (Table 2). An additional analysis found that a significantly higher percentage of patients aged $\leq 50$ years had functional catheter malfunctions caused by omentum wrap than did patients aged $>50$ years $(21.7 \%$ vs. $7.3 \%, P=0.039)$.

Prevalence of other urgent-start PD related complications For perioperative complications, significant hemorrhage presented in $3(0.1 \%)$ patients and none suffered bowel perforation. Early peritonitis occurred in 24 patients (0.28 per patient-year), and early exit-site infections occurred in 7 patients ( 0.08 per patient-year). Details of the relevant organisms and outcomes of these episodes are provided in the additional file (Additional file 1: Table S1 and Table S2). For the 147 patients (7.1\%) who experienced abdominal wall complications (including hernia, hydrothorax, hydrocele, pericatheter leakage, and subcutaneous leakage), 36 (24.5\%) of these events happened within the first month after catheter insertion. Among the early-PD abdominal wall complications, pericatheter leakage was the most common complication, which responded well to conventional treatments; none resulted in technique failure. By contrast, hernia, hydrothorax, and hydrocele were the most common major complications of late-stage PD. Patients with hydrothorax were transferred to HD (Table 3). After

Table 2 Risk factors for functional catheter problem and catheter failure in COX models

\begin{tabular}{|c|c|c|c|c|}
\hline \multirow[t]{2}{*}{ Variables } & \multicolumn{2}{|l|}{ Univariate model } & \multicolumn{2}{|c|}{ Multivariate model } \\
\hline & $\mathrm{HR}(95 \% \mathrm{Cl})$ & $P$ value & $\mathrm{HR}(95 \% \mathrm{Cl})$ & $P$ value \\
\hline \multicolumn{5}{|l|}{ Functional catheter problem } \\
\hline Age (every 5 years increase) & $0.86(0.81-0.91)$ & $<0.001$ & $0.86(0.81-0.92)$ & $<0.001$ \\
\hline Sex (Male) & $1.08(0.79-1.49)$ & 0.623 & $1.01(0.72-1.44)$ & 0.942 \\
\hline BMI $\left(\mathrm{kg} / \mathrm{m}^{2}\right)$ & $0.94(0.89-0.99)$ & 0.024 & $0.97(0.92-1.03)$ & 0.266 \\
\hline Serum creatinine (mg/dL) & $1.04(1.00-1.08)$ & 0.023 & $1.03(0.99-1.06)$ & 0.934 \\
\hline Hemoglobin (g/dL) & $0.92(0.84-1.01)$ & 0.068 & $0.96(0.88-1.03)$ & 0.346 \\
\hline $\mathrm{BSA}\left(\mathrm{m}^{2}\right)$ & $0.66(0.25-1.77)$ & 0.410 & - & - \\
\hline Diabetic nephropathy & $0.83(0.55-1.24)$ & 0.359 & - & - \\
\hline History of abdominal surgery & $1.38(0.81-2.35)$ & 0.235 & - & - \\
\hline eGFR $\left(\mathrm{ml} / \mathrm{min} / 1.73 \mathrm{~m}^{2}\right)$ & $0.96(0.89-1.03)$ & 0.227 & - & - \\
\hline Serum albumin (g/dL) & $0.89(0.65-1.23)$ & 0.482 & - & - \\
\hline \multicolumn{5}{|l|}{ Catheter failure } \\
\hline Age (every 5 years increase) & $0.80(0.74-0.87)$ & $<0.001$ & $0.81(0.73-0.89)$ & $<0.001$ \\
\hline Sex (Male) & $1.06(0.67-1.68)$ & 0.817 & $1.25(0.75-2.10)$ & 0.393 \\
\hline BMI $\left(\mathrm{kg} / \mathrm{m}^{2}\right)$ & $0.91(0.85-0.98)$ & 0.017 & $0.94(0.86-1.03)$ & 0.164 \\
\hline Serum creatinine (mg/dL) & $1.07(1.02-1.12)$ & 0.008 & $1.05(0.98-1.10)$ & 0.765 \\
\hline Hemoglobin (g/dL) & $0.90(0.79-1.03)$ & 0.133 & $0.92(0.81-1.04)$ & 0.364 \\
\hline $\mathrm{BSA}\left(\mathrm{m}^{2}\right)$ & $0.42(0.10-1.74)$ & 0.234 & - & - \\
\hline Diabetic nephropathy & $0.83(0.46-1.48)$ & 0.517 & - & - \\
\hline History of abdominal surgery & $1.55(0.75-3.23)$ & 0.241 & - & - \\
\hline eGFR $\left(\mathrm{ml} / \mathrm{min} / 1.73 \mathrm{~m}^{2}\right)$ & $0.96(0.87-1.07)$ & 0.468 & - & - \\
\hline Serum albumin (g/dL) & $0.97(0.61-1.54)$ & 0.886 & - & - \\
\hline
\end{tabular}


Table 3 Other complications related to urgent-start PD and outcomes in the entire cohort $(n=2059)$

\begin{tabular}{|c|c|c|c|c|}
\hline Complications & Overall (n) & Early present $(n)^{c}$ & Surgical Repair(n $)^{d}$ & Result in technique failure $(n)^{d}$ \\
\hline \multicolumn{5}{|l|}{ Perioperative complications } \\
\hline Bowel perforation & 0 & 0 & 0 & 0 \\
\hline Significant hemorrhage ${ }^{a}$ & $3(0.1 \%)$ & $3(0.1 \%)$ & 2 & 0 \\
\hline Early infections ${ }^{\mathrm{b}}$ & $31(1.5 \%)$ & - & - & - \\
\hline Peritonitis & $24(1.2 \%)$ & - & - & - \\
\hline Exit-site infections & $7(0.3 \%)$ & - & - & - \\
\hline \multicolumn{5}{|l|}{ Abdominal wall complications } \\
\hline Hernia & $74(3.6 \%)$ & $4(0.2 \%)$ & $18(24.3 \%)$ & $8(10.8 \%)$ \\
\hline Inguinal hernia & $34(1.7 \%)$ & $3(0.1 \%)$ & $15(44.1 \%)$ & $5(14.7 \%)$ \\
\hline Umbilical hernia & $40(1.9 \%)$ & $1(0.05 \%)$ & $3(7.5 \%)$ & $3(7.5 \%)$ \\
\hline Hydrothorax & $18(0.9 \%)$ & $3(0.1 \%)$ & 0 & $18(100 \%)$ \\
\hline Hydrocele & $29(1.4 \%)$ & $8(0.4 \%)$ & $4(13.8 \%)$ & $9(31.0 \%)$ \\
\hline Pericatheter leakage & $19(0.9 \%)$ & $19(0.9 \%)$ & 0 & 0 \\
\hline Subcutaneous leakage & $7(0.3 \%)$ & $2(0.1 \%)$ & 0 & $4(57.1 \%)$ \\
\hline
\end{tabular}

Note. ${ }^{a}$ Hemorrhage requiring transfusion or surgical intervention;

${ }^{b}$ Peritonitis and exit-site infections within 2 weeks after catheter insertion;

c Present within the first month;

${ }^{d}$ The percentage in each subtype of complications

adjusting for confounders in multivariate analyses, male sex (HR: 1.43, 95\%CI: 1.00-2.04), diabetic nephropathy (HR: 1.56, 95\%CI: 1.08-2.25), and lower hemoglobin levels (HR:0.89, 95\%CI: 0.81-0.98) were independent risk factors for abdominal wall complications (Table 4).

\section{Overall technique survival}

During the follow-up period, 291 patients (14.1\%) were transferred to HD, 430 (20.9\%) received renal transplants, $534(25.9 \%)$ died, and $738(35.8 \%)$ remained on PD. The crude death-censoring technique failure rate was 0.04 per patient-year. Kaplan-Meier analysis showed that at the end of 1-month, 1-year, 3-year, 5-year, and 10-year, technique survival rate was 99.5, 97.0, 90.3, 82.7, and 58.8\%, respectively (Fig. 5).

\section{Discussion}

In the present cohort study of 2059 ESRD patients received urgent-start PD, their short-term and long-term rates for catheter failure and other catheter-related complications (such as bowel perforation, significant hemorrhage, early infections, and abdominal wall complications) were very low. Catheter patency rates were 1-month: $97.6 \%, 1$ year: 96.4\%, 3-year: $96.2 \%$ and 5-year: 96.2\%; while technique survival rates were 1-month: 99.5\%, 1-year: 97.0\%, 3-year: $90.3 \%$, and 5-year: $82.7 \%$.

Table 4 Risk factors for abdominal wall complications in COX models

\begin{tabular}{|c|c|c|c|c|}
\hline \multirow[t]{2}{*}{ Variables } & \multicolumn{2}{|l|}{ Univariate model } & \multicolumn{2}{|c|}{ Multivariate model } \\
\hline & $\mathrm{HR}(95 \% \mathrm{Cl})$ & $P$ value & HR $(95 \% \mathrm{Cl})$ & $P$ value \\
\hline Age (every 5 years increase) & $0.99(0.95-1.06)$ & 0.985 & $1.00(0.95-1.06)$ & 0.944 \\
\hline Sex (Male) & $1.39(0.98-1.97)$ & 0.069 & $1.43(1.00-2.04)$ & 0.048 \\
\hline Diabetic nephropathy & $1.59(1.10-2.30)$ & 0.013 & $1.56(1.08-2.25)$ & 0.017 \\
\hline History of abdominal surgery & $0.51(0.21-1.24)$ & 0.136 & $0.50(0.21-1.23)$ & 0.133 \\
\hline Hemoglobin (g/dL) & $0.90(0.82-0.99)$ & 0.027 & $0.89(0.81-0.98)$ & 0.016 \\
\hline $\mathrm{BMI}\left(\mathrm{kg} / \mathrm{m}^{2}\right)$ & $1.02(0.97-1.08)$ & 0.436 & - & - \\
\hline Type of catheter (coiled VS. straingt) & $1.03(0.56-1.92)$ & 0.916 & - & - \\
\hline Serum creatinine (mg/dL) & $1.01(0.97-1.05)$ & 0.665 & - & - \\
\hline $\mathrm{BSA}\left(\mathrm{m}^{2}\right)$ & $1.71(0.60-4.89)$ & 0.315 & - & - \\
\hline $\mathrm{eGFR}\left(\mathrm{ml} / \mathrm{min} / 1.73 \mathrm{~m}^{2}\right)$ & $1.05(0.94-1.08)$ & 0.891 & - & - \\
\hline Serum albumin (g/dL) & $0.86(0.61-1.21)$ & 0.379 & - & - \\
\hline
\end{tabular}

Abbreviations: BMI body mass index, BSA body surface area, eGFR estimated glomerular filtration rate, $H R$ hazard ratio, $C l$ confidence interval 


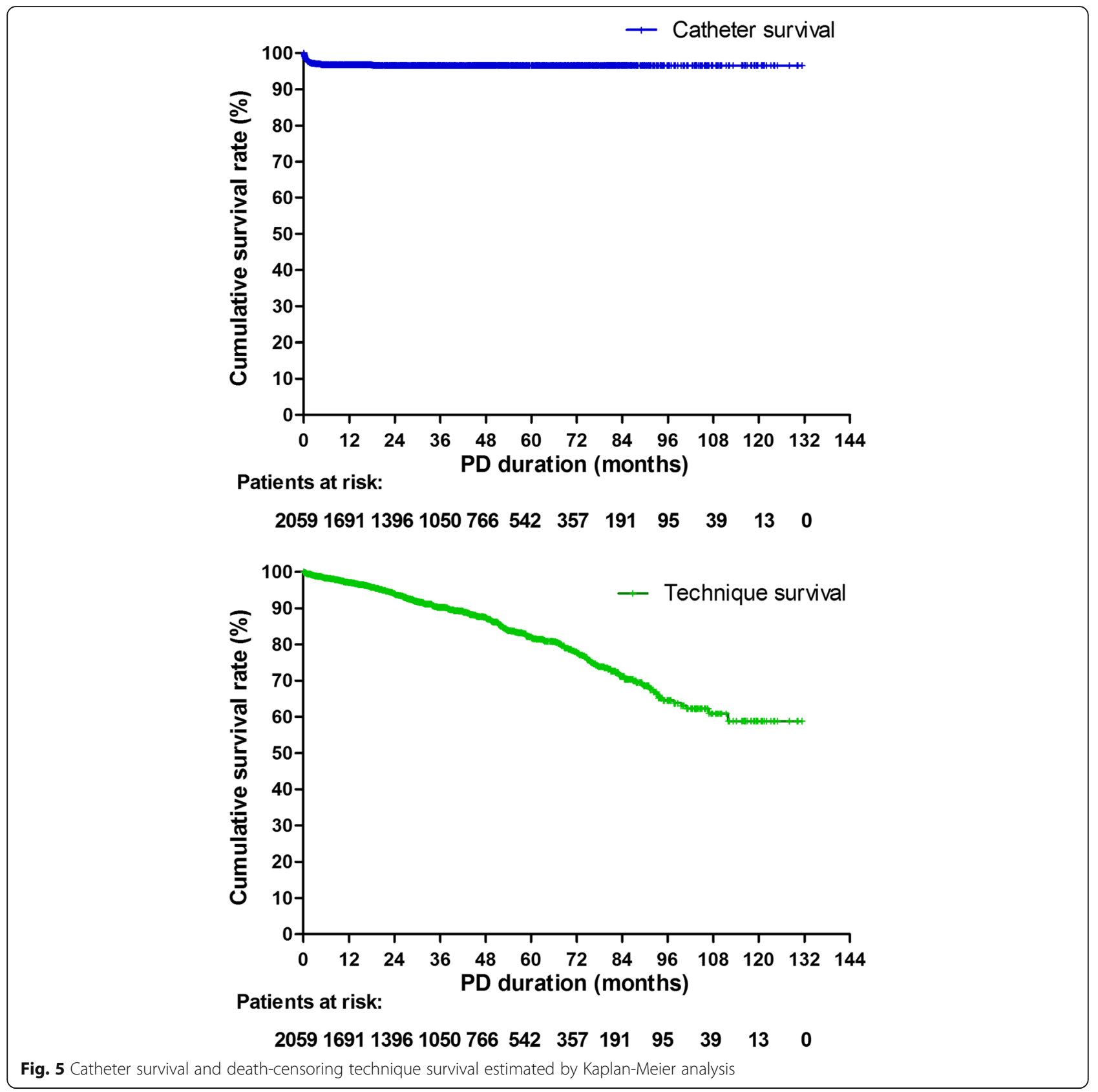

Catheter patency has been emphasized as the primary marker of successful PD catheter insertion; and ISPD guidelines recommend that the rate should be $>80 \%$ at 1 year [9]. Low catheter patency is a major concern in urgent-start PD because earlier-start PD may irritate the omentum and cause omental wrapping, thus leading to the catheter failure $[9,11,21]$. However, catheter patency rates were rarely reported in previous studies on urgent-start PD, whereas functional catheter problems (including catheter malfunction, catheter shift, and/or omental wrapping) reportedly fluctuated between 2.0 and $15.4 \%$ in previous studies $[5,11,13,14,16]$. In our study, 156 (7.6\%) patients experienced functional catheter problems; $40.4 \%$ of their functional catheter problems occurred within 14 days after insertion, and 51.9\% were rescued by the conservative methods. Only $3.6 \%$ of the catheters in our study cohort failed and needed surgical interventions. The 1-year catheter patency rate and technique survival rate in our study population was far in excess of the recommended target $[9,22]$. By our experience, careful feedback and communication among the nephrologists, the PD nurses and the patients was helpful for promptly catching and solving functional catheter problems. We have a team with excellent 
nephrologists and nurses who responsible for the process of PD therapy from inpatient to outpatient treatment. Generally, catheter-related complications would be estimated by the PD nurses firstly and then diagnosed by nephrologists. The team would discuss whether the complications were related to catheter insertion technique, or insufficient training, or other causes. Finally, some potentially useful suggestions to solve the problems would be proposed. In addition, early initiation of low-volume PD may also help prevent catheter obstructions, and local adhesions which could eventually lead to catheter failure [23]. We also found that younger age was a risk factor independently associated with higher catheter failure in the current study. Further result showed that younger patients had higher percentages of omentum wrapping as a cause of functional catheter malfunctions. Perhaps younger patients have more active omentum, which may lead to a higher rate of omentum wrapping and subsequent catheter failure.

Abdominal wall complications (especially pericatheter leakage, which happens early after catheter insertion) are also major concerns in urgent-start PD [9]. Reported incidence of dialysate leakage varies from 1.5 to $37 \%$ in regular PD patients [5, 24-27], but fluctuates between 0 and 13.5\% in patients who receive urgent-start PD [5, 11, 13-16]. In theory, increased intraperitoneal infusion during PD will increase the intraperitoneal pressure, and eventually increase the risk of developing abdominal wall complications. Our results shown that early pericatheter leakage only occurred in $19(0.9 \%)$ patients in our cohort, and all these cases responded well to conservative treatments; none of them resulted in technique failure. As introduced in the present study, a double purse-string suture on the posterior rectus sheath and parietal peritoneum (Fig. 2) was used to fix the catheter and strengthen the "artificial weakness" at the catheter insertion points. In addition, a gradually increasing IPD regimen (Fig. 1) to start PD was used in the current study to prevent suddenly increased intraperitoneal pressure. The lower intra-abdominal pressure could prevent dialysate leaks and allow abdominal incisions time to heal after catheter insertion. In this way, the incidence of pericatheter leakage was successfully decreased. These results suggest that urgent-start peritoneal dialysis can be effectively conducted with an acceptable rate of abdominal wall complications. Our study also identified that male sex, diabetic nephropathy and lower hemoglobin levels were independent risk factors for abdominal wall complications, which indicates that we should pay more attention to these patients at risk for assessment and management before initiating PD, and during follow-up period.

Early infection is another concern about urgent-start peritoneal dialysis. A Danish study reported that whereas $15.4 \%$ of urgent-start peritoneal dialysis patients suffered peritonitis within the first 3 months, $15.4 \%$ of the planned PD group also suffered peritonitis [11]. However, in the present study, early peritonitis occurred only in $1.2 \%$ of our patients, and the long-term overall incidence of peritonitis was 0.16 cases per patient-year, as reported in our previous study [28]. In our center, a single-dose of prophylactic antibiotic was given $30 \mathrm{~min}$ before catheterization. All incident PD patients and their caregivers would undergo standard PD training. Taken together, strict sterilization procedure, preoperative antibiotic prophylaxis, experienced nephrologists for catheter insertion, and a professional nursing team to train patients and caregivers may help control occurrence of early peritonitis.

Several limitations should be taken into consideration in this study. First, selection bias was inevitable for a singlecenter cohort study. Second, as a retrospective study, we could not control some potential confounders, such as variations among catheter insertion techniques by different operators. Third, as we lacked a control group, our study still cannot show whether urgent-start peritoneal dialysis is better or inferior to planned PD for patients who can wait for planned PD without receiving HD.

\section{Conclusion}

Our results over 10 years show that ESRD patients who received urgent-start peritoneal dialysis had high rates of catheter patency and technique survival, and low incidences of catheter-related complications, which indicates that urgent-start peritoneal dialysis is safe, efficacious, and practical for patients with ESRD. A unique infrastructure and management approach that includes a well-trained PD team, a standardized procedure for catheter insertion by experienced nephrologists, a carefully designed initial PD prescription, a well-planned training curriculum for patients and their caregivers, and comprehensive followup care appear to be essential for the successful urgentstart peritoneal dialysis program. Clinical outcomes of unplanned versus planned dialysis starters among different PD centers warrant prospective study.

\section{Additional files}

Additional file 1: Table S1. Details of the organisms and the outcomes of the eraly peritonitis. Table $\mathbf{S 2}$. Details of the organisms and the outcomes of the early exit-site infections. (DOCX $13 \mathrm{~kb}$ )

\section{Abbreviations}

BMI: Body mass index; BSA: Body surface area; CAPD: Continuous ambulatory peritoneal dialysis; CKD: Chronic kidney disease; ESRD: End stage renal disease; HD: Hemodialysis; HR: Hazard ratio; IPD: Intermittent peritoneal dialysis; IQR: Interquartile range; PD: Peritoneal dialysis; SD: Standard deviation; USRDS: United States Renal Data System

\section{Acknowledgements}

We thank all doctors, nurses and patients in our PD center, for their efforts for the current study. We thank Marla Brunker, from Liwen Bianji, Edanz 
Group China (https://www.liwenbianji.cn), for editing the English text of a draft of this manuscript.

\section{Authors' contributions}

HY and XY1 contributed equally to this work. They proposed the conception, designed the work, did the acquisition, analysis and interpretation of data for the work. They agreed to be accountable for all aspects of the work. CY, QG, YL, QY, WC, HM, JL1, YQ, XZ, DZ, JL2, ZL, ZJ, and FH, provided substantial contribution to the acquisition, analysis and interpretation of data for the work, drafting of the manuscript and agreement to be accountable for all aspects of the work. $X Y 1$ and $X Y 2$ are the senior authors and main contributor to the conception and design, analysis and interpretation of data for the work, drafting of the work, and agreement to be accountable for all aspects of the work. All authors have read and approve of the final version.

\section{Funding}

This work was supported by the Natural Science Foundation of China (Grant no. 81774069, 81570614), National Key Research and Development Program (Grant no. 2016YFC0906101), the Guangdong Science Foundation of China (Grant no. 2014B020212020, 2017A050503003, 2017B020227006), Foundation of Guangdong Key Laboratory of Nephrology (Grant no. 2017B030314019), and the Guangzhou Committee of Science and Technology, China (Grant no. 2014Y2-00543, 201704020167), and Science and Technology Planning Project of Guangdong Province of China (Grant no. A2018353). These funding supported the the collection, management, and analysis of data, as well as manuscript editing and article-processing charge for publish.

\section{Availability of data and materials}

The datasets used and/or analyzed during the current study are available from the corresponding author on reasonable request.

\section{Ethics approval and consent to participate}

The study protocol was consistent with the ethical principles of the Helsink Declaration and was approved by the Ethics Committee of The First Affiliated Hospital, Sun Yat-sen University. All participants were asked for permission to use their medical data for a non-commercial study, and written informed consent was obtained from them at the initiation of PD follow-up.

\section{Consent for publication}

Not applicable.

\section{Competing interests}

The authors declare that they have no competing interests.

Received: 26 April 2018 Accepted: 3 June 2019

Published online: 02 July 2019

\section{References}

1. United States Renal Data System. 2017 USRDS Annual Data Report: Epidemiology of kidney disease in the United States. Bethesda: National Institutes of Health, National Institute of Diabetes and Digestive and Kidney Diseases; 2017

2. Yu X. How to set up PD centres: the Chinese perspective. 2017. http://www. thelancet.com/campaigns/kidney/updates. Accessed May 2017.

3. Zhang L, Wang F, Wang L, Wang W, Liu B, Liu J, et al. Prevalence of chronic kidney disease in China: a cross-sectional survey. Lancet. 2012;379(9818):815-22.

4. Xu R, Han QF, Zhu TY, Ren YP, Chen JH, Zhao HP, et al. Impact of individual and environmental socioeconomic status on peritoneal dialysis outcomes: a retrospective multicenter cohort study. PLoS One. 2012;7(11):e50766.

5. Xu D, Liu T, Dong J. Urgent-start peritoneal Dialysis complications: prevalence and risk factors. Am J Kidney Dis. 2017;70(1):102-10.

6. Yu X, Yang X. Peritoneal dialysis in China: meeting the challenge of chronic kidney failure. Am J Kidney Dis. 2015;65(1):147-51.

7. Ministry of Health, P. R. China: Chinese National Renal Data System. (online) http://wwwenrdsnet 2017.

8. Dombros N, Dratwa M, Feriani M, Gokal R, Heimburger O, Krediet R, et al. European best practice guidelines for peritoneal dialysis. 3 peritoneal access. Nephrol Dial Transplant. 2005;20(Suppl 9):i8-i12.

9. Figueiredo A, Goh BL, Jenkins S, Johnson DW, Mactier R, Ramalakshmi S, et al. Clinical practice guidelines for peritoneal access. Perit Dial Int. 2010;30(4):424-9.
10. Alkatheeri AM, Blake PG, Gray D, Jain AK. Success of urgent-start peritonea Dialysis in a large Canadian renal program. Perit Dial Int. 2016;36(2):171-6.

11. Povlsen $J V$, Ivarsen P. How to start the late referred ESRD patient urgently on chronic APD. Nephrol Dial Transplant. 2006;21(Suppl 2):ii56-9.

12. See EJ, Cho Y, Hawley CM, Jaffrey LR, Johnson DW. Early and late patient outcomes in urgent-start peritoneal Dialysis. Perit Dial Int. 2017:37(4):414-9.

13. Reyes-Marín FA, Gómez-Villanueva D, Ballesteros-Santiago A, Amato D. Urgent peritoneal Dialysis initiation: is it better to wait a few days than to use the catheter immediately after its implantation? A randomized controlled trial. Intern Med. 2014;4(4):1000159.

14. Masseur A, Guest S, Kumar V. Early technique success after initiation of treatment with urgent-start peritoneal dialysis. Adv Perit Dial. 2014;30:36-9.

15. Koch M, Kohnle M, Trapp R, Haastert B, Rump LC, Aker S. Comparable outcome of acute unplanned peritoneal dialysis and haemodialysis. Nephrol Dial Transplant. 2012;27(1):375-80.

16. Yang YF, Wang HJ, Yeh CC, Lin HH, Huang CC. Early initiation of continuous ambulatory peritoneal dialysis in patients undergoing surgical implantation of Tenckhoff catheters. Perit Dial Int. 2011;31(5):551-7.

17. Yi C, Guo Q, Lin J, Huang F, Yu X, Yang X. Clinical outcomes of remote peritoneal Dialysis patients: a retrospective cohort study from a single Center in China. Blood Purif. 2016;41(1-3):100-7.

18. Levey AS, Stevens LA, Schmid CH, Zhang YL, Castro AF 3rd, Feldman $\mathrm{HI}$, et al. A new equation to estimate glomerular filtration rate. Ann Intern Med. 2009:150(9):604-12.

19. Yang X, Yi C, Liu X, Guo Q, Yang R, Cao P, et al. Clinical outcome and risk factors for mortality in Chinese patients with diabetes on peritoneal dialysis: a 5-year clinical cohort study. Diabetes Res Clin Pract. 2013;100(3):354-61.

20. Bammens B, Peeters D, Jaekers J, Claes KJ, Evenepoel P, Kuypers D, et al. Postimplantation $\mathrm{X}$-ray parameters predict functional catheter problems in peritoneal dialysis. Kidney Int. 2014;86(5):1001-6.

21. Liu Y, Zhang L, Lin A, Ni Z, Qian J, Fang W. Impact of break-in period on the short-term outcomes of patients started on peritoneal dialysis. Perit Dial Int. 2014:34(1):49-56.

22. McCormick BB, Bargman JM. Noninfectious complications of peritoneal dialysis: implications for patient and technique survival. J Am Soc Nephrol. 2007;18(12):3023-5.

23. Gadallah MF, Torres-Rivera C, Ramdeen G, Myrick S, Habashi S, Andrews G. Relationship between intraperitoneal bleeding, adhesions, and peritoneal dialysis catheter failure: a method of prevention. Adv Perit Dial. 2001;17:127-9.

24. Del Peso G, Bajo MA, Costero O, Hevia C, Gil F, Diaz C, et al. Risk factors for abdominal wall complications in peritoneal dialysis patients. Perit Dial Int. 2003:23(3):249-54

25. Song JH, Kim GA, Lee SW, Kim MJ. Clinical outcomes of immediate fullvolume exchange one year after peritoneal catheter implantation for CAPD. Perit Dial Int. 2000;20(2):194-9.

26. Lam MF, Lo WK, Tse KC, Yip TP, Lui SL, Chan TM, et al. Retroperitoneal leakage as a cause of acute ultrafiltration failure: its associated risk factors in peritoneal dialysis. Perit Dial Int. 2009:29(5):542-7.

27. Leblanc M, Ouimet D, Pichette V. Dialysate leaks in peritoneal dialysis. Semin Dial. 2001:14(1):50-4.

28. Ye H, Zhou Q, Fan L, Guo Q, Mao H, Huang F, et al. The impact of peritoneal dialysis-related peritonitis on mortality in peritoneal dialysis patients. BMC Nephrol. 2017;18(1):186.

\section{Publisher's Note}

Springer Nature remains neutral with regard to jurisdictional claims in published maps and institutional affiliations.

Ready to submit your research? Choose BMC and benefit from

- fast, convenient online submission

- thorough peer review by experienced researchers in your field

- rapid publication on acceptance

- support for research data, including large and complex data types

- gold Open Access which fosters wider collaboration and increased citations

- maximum visibility for your research: over $100 \mathrm{M}$ website views per year

At BMC, research is always in progress.

Learn more biomedcentral.com/submission 